\title{
Pseudoexfoliative disease of the lens: a study in electron microscopy and histochemistry
}

\author{
A. J. DARK, B. W. STREETEN, AND C. C. CORNWALl \\ From the Departments of Ophthalmology and Pathology, Veterans Administration Hospital \\ and State University of New York, Upstate Medical Center, Syracuse
}

SUMMARY The anterior lens capsule in pseudoexfoliative (PE) disease has been investigated with scanning electron microscopy and transmission electron microscopy. It shows several distinct zones. which correlate with those seen clinically. True exfoliation of the anterior lens capsule is shown as an integral pathologic feature of PE disease.

PE fibrils are mixed with various degraded cell products, including organelles, of which uveal pigment granules are the most frequent. Sheets of degenerating iris pigment epithelium as well as individual cells are often firmly adherent to the pre-equatorial lens capsule and zonules.

PE fibrils on the capsule are 35 to $40 \mathrm{~nm}$ in width, often showing several subunit filaments about $10 \mathrm{~nm}$ wide. PE filaments forming vertical bundles in the pre-equatorial capsule are $10 \mathrm{~nm}$ wide, but show little tendency to aggregate laterally. Histochemical and ultrastructural properties of both types of PE fibril and those of the capsular inclusions suggest that they are closely related fibrillar proteins. PE fibrils share some of the characteristics of amyloid but are not identical substances. Nevertheless, a similar mechanism of synthesis is suggested. The participation of non-lenticular sites in the formation of PE fibrils is not precluded by this hypothesis.

Pseudoexfoliative (PE) disease of the lens, a disorder of widespread geographic and racial distribution, is of unknown aetiology. It has been demonstrated in the Bantu tribe in South Africa that a subtle radial striation of the anterior lens capsule, occurring in early or middle adult life, represents an early slitlamp sign of this disease (Bartholomew, 1971), but the classical manifestations are almost exclusively geriatric. Vogt (1925) believed that the greyish frosting material lying on the anterior lens surface and on other structures bathed in aqueous was produced by vacuolation and exfoliation of the superficial capsule. A similar mechanism involving the zonular lamella has been proposed by Gifford (1957) and more recently by Sugar (1976). Ashton et al. (1975), however, have noted that there is no transmission electron micrographic (TEM) evidence for the existence of a zonular lamella except in the peripheral region of the lens, where the zonules insert. The concept of an exfoliative process is nevertheless strongly supported by slit-lamp microscopy, which often shows the PE material to be flaking or rolling

Address for reprints: Dr A. J. Dark, Eye Department, Veterans Administration Hospital, Syracuse, New York 13210, USA up as a layer central to the zonular insertion. In contrast, PE material in sections stained in haematoxylin and eosin is seen usually as discrete, pink bush-like masses attached in either a sessile or pedunculated manner to the anterior lens surface but often without evidence of capsular dehiscence. This appearance led Busacca (1928) to interpret them as deposits of an unidentified material of unknown origin. An attempt to synthesise these somewhat divergent observations and to distinguish the disorder from true (thermal) exfoliation of the lens. capsule resulted in the introduction by DvorakTheobold (1954) of the semantically confusing name 'pseudoexfoliation', which is in general use.

The recent (TEM) studies of Ghosh and Speakman (1972) have indicated that a true shedding of the superficial capsule may indeed occur. Davanger and Pederson (1975) and Davanger (1975) using TEM and scanning electron microscopy (SEM) have likewise shown that the PE excrescences rest on a 'basal lamina', which has a marked tendency to. curl up.

The cells which synthesise PE material have not yet been identified in spite of several transmission electron microscopy studies involving the anterior segment of the eye. Thus, Ashton et al. (1965), who. 
examined lens, iris, and ciliary body in PE disease, were unable to determine a site of origin. They did note, however, a pathognomonic feature of this disorder, namely, the presence of PE-like material in the deep layer of the lens capsule immediately anterior to the equator. This deep layer was detected independently by Bertelsen et al. (1964), who also observed that it was often vertically striated under the light microscope. TEM showed that the striations correspond with bundles of fibrillar material located in deep pits of the adjacent anterior lens epithelium. This latter appearance persuaded these authors that the pre-equatorial zone of the anterior lens epithelium is a primary site for the production of PE material.

The pre-equatorial zone of the normal lens capsule contains numerous inclusions which have been shown by Bertelsen and Ehlers (1969) and Dark et al. (1969) to have TEM and histochemical properties similar to PE fibrils. Capsular inclusions are unduly elongated and present in profusion in PE disease. These and other observations led Dark et al. (1969) to postulate that the lens production of capsular inclusions is accelerated in some way in PE disease, and that a similar process leads to synthesis of PE fibrils both within and outside the lens capsule. Ghosh and Speakman $(1973,1974)$ in TEM studies noted distinctive changes in the epithelia of the iris and ciliary body which led them to propose these as additional primary sites of PE fibril manufacture.

Ringvold (1973a) observed that PE material was also deposited in the walls of conjunctival capillaries, a finding confirmed and extended by Ghosh and Speakman (1976). Using iris fluorescence angiography, Vannas (1969) demonstrated abnormal vessels leaking fluorescein. Hence it was suggested that PE material is blood-borne, diffusing into the anterior chamber through walls of diseased vessels of the iris.

The present study is an attempt to clarify the SEM and TEM correlates of well-known clinical observations. At the same time the alleged role of the pre-equatorial lens epithelial cells in the pathogenesis of PE disease is evaluated further.

\section{Methods and materials}

SCANNING ELECTRON MICROSCOPY

Six cataractous lenses were obtained from 5 septuagenarian patients who had normal ocular tensions. All were studied by slit-lamp microscopy after mydriasis. Patient 1 had unilateral PE disease. Both lenses were obtained, the apparently unaffected lens to act as control. Patients 2, 3, and 4, who had bilateral PE disease, provided another 3 lenses. The sixth lens, from Patient 5, clinically unaffected with PE disease, provided a further control.

Lenses were extracted with a cryoprobe without using alpha-chymotrypsin. They were fixed in cold, phosphate-buffered $2.5 \%$ glutaraldehyde for 1 hour, then osmified before dehydrating in graduated acetones. Finally, absolute acetone was removed by critical point drying with liquid carbon dioxide. The desiccated lenses were then vacuum-coated with carbon followed by gold-palladium before examination in an ETEC Autoscan scanning electron microscope which had been previously calibrated.

TRANSMISSION ELECTRON MICROSCOPY Eight cataractous lenses from patients afflicted with PE disease were obtained at operation. Three lenses were immediately fixed in phosphate-buffered $2.5 \%$ glutaraldehyde for 2 hours. Osmification followed, and then small pieces of anterior lens capsule were excised from the various zones before embedding in epoxy resin. These sections were stained with uranyl acetate and lead citrate before examination under an RCA transmission electron microscope.

\section{HISTOCHEMISTRY}

Three cataractous lenses affected with PE disease were fixed in formalin and then embedded in paraffin. Sections from these lenses were stained with haematoxylin and eosin and a number of special stains to be mentioned alongside the results. Two unfixed cataractous lenses showing typical clinical features of PE disease were cut frozen, and the sections were stained with fluorescein antibody conjugates for lambda and kappa immunoglobulin light chains.

\section{Observations}

SCANNING ELECTRON MICROSCOPY

Apart from zonular remnants the anterior surfaces of the 2 clinically normal lenses have a faint granular texture at high magnification.

The 4 lenses clinically affected with PE disease were similar in general appearance and are described together. The general topography of the anterior lens surface in PE disease can be best seen at low magnification (Fig. 1). The various zones correlate well with the appearances of slit-lamp microscopy, showing a greyish central zone separated by a featureless intermediate annulus from the region of granular deposits, then a peripheral zone, and finally a pre-equatorial zone. The central zone comprises a layer of fibrils (40 to $60 \mathrm{~nm}$ in diameter) in which are embedded numerous smooth-surfaced ovoid bodies. The ovoid bodies are orientated vertically and vary in height from 0.5 to $1.0 \mu \mathrm{m}$ and 


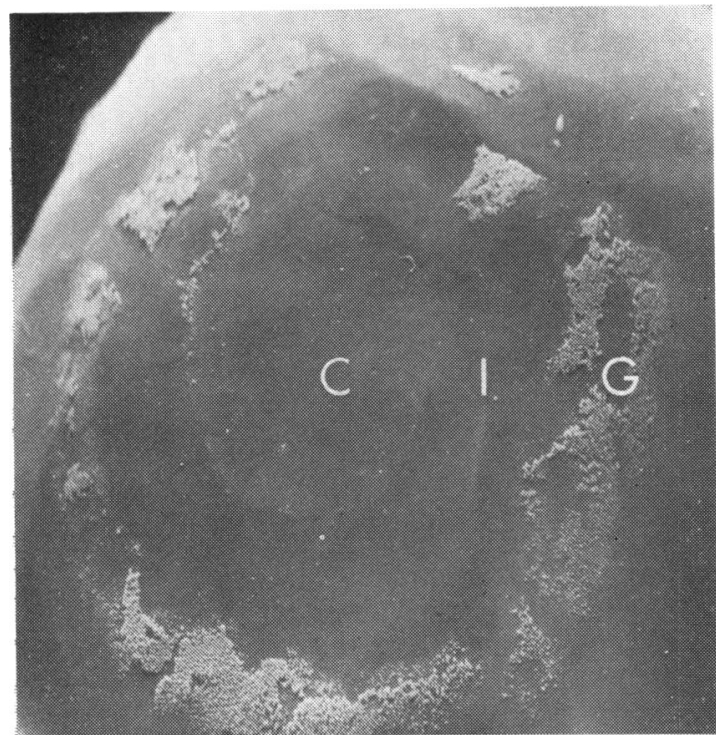

Fig. 1 Anterior surface of the lens in PE disease, illustrating central zone (C), intermediate zone (I) and the granular zone $(\mathrm{G})$. $\quad S E M \times 7$

are 0.25 to $0.50 \mu \mathrm{m}$ wide (Fig. 2). At the periphery of the central zone are variable heaped-up aggregates of fibrillar material. Similar but sparser elements are found in the intermediate zone. The granular zone, at low magnification, is seen as a grove of vegetations (Fig. 3 inset) closely grouped together.
The largest vegetations $(15 \times 15 \mu \mathrm{m})$ are found in the middle of this zone with a gradual decrease in size towards the edges, where the smallest $(5 \times 3 \mu \mathrm{m})$ are found. The vegetations, which are occasionally joined together by a few fibrils 40 to $60 \mathrm{~nm}$ wide (Fig. 3), are for the most part discrete. Each comprises a skein of closely-knit fibrils interspersed with numerous ovoid bodies; these components have similar dimensions to those in the intermediate and central zones. The lens surface in this zone, where it can be viewed, is overlaid with occasional fibrils on which ovoid bodies can be seen usually singly but sometimes grouped. The rolled appearance of the inner edge of this zone is produced by a peeling and curling up of the surface layer, to which the vegetations are attached (Fig. 4).

Here and there vegetations similar to those seen in the granular zone encrust occasional zonular remnants.

\section{TRANSMISSION ELECTRON MICROSCOPY}

Central Zone.-Sections from the central zone show that the superficial layer of the capsule has a somewhat denser structure than the main part. This surface zone is often separated by a rarefied seam from the main bulk of the underlying capsule. Its attachment is evidently tenuous, so that in places it is dehiscent and rolled up. Embedded in this surface layer are a few membrane-clad organelles, most of which are clearly identifiable as pigment granules. Occasional mounds of unidentified amorphous

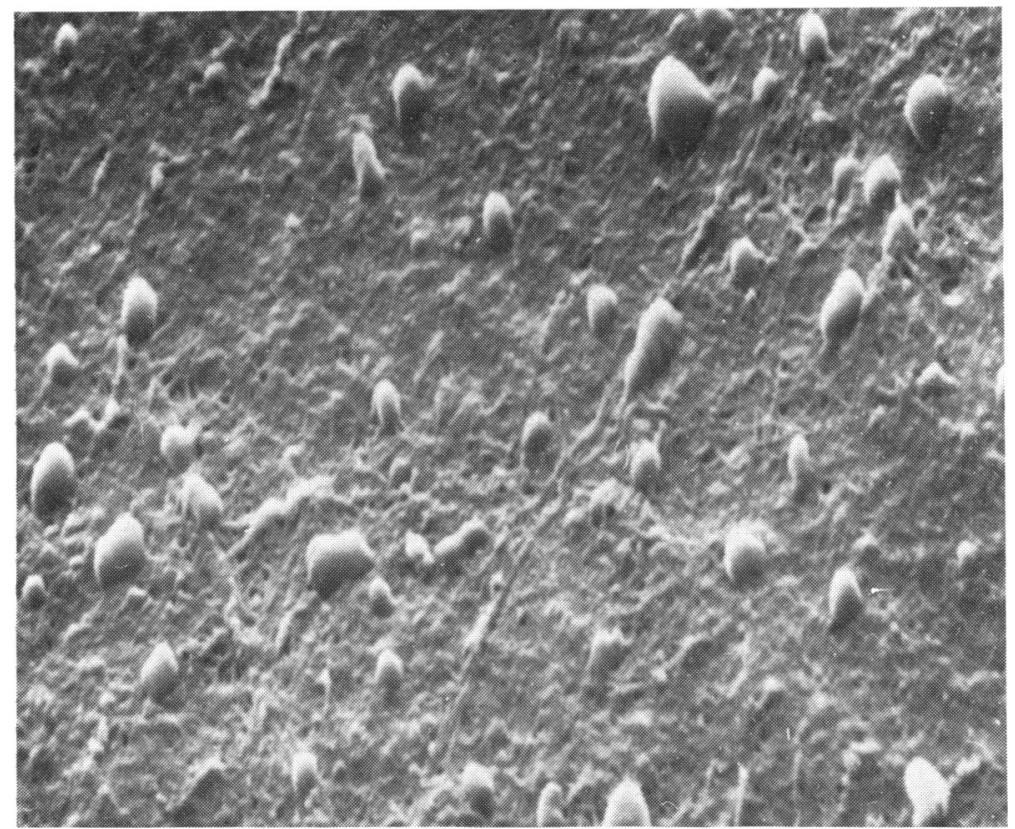

Fig. 2 Anterior surface of the lens in PE disease. Central zone depicting meshwork of fibrils with embedded ovoid bodies. SEM $\times \mathbf{3 5 5 0}$ 


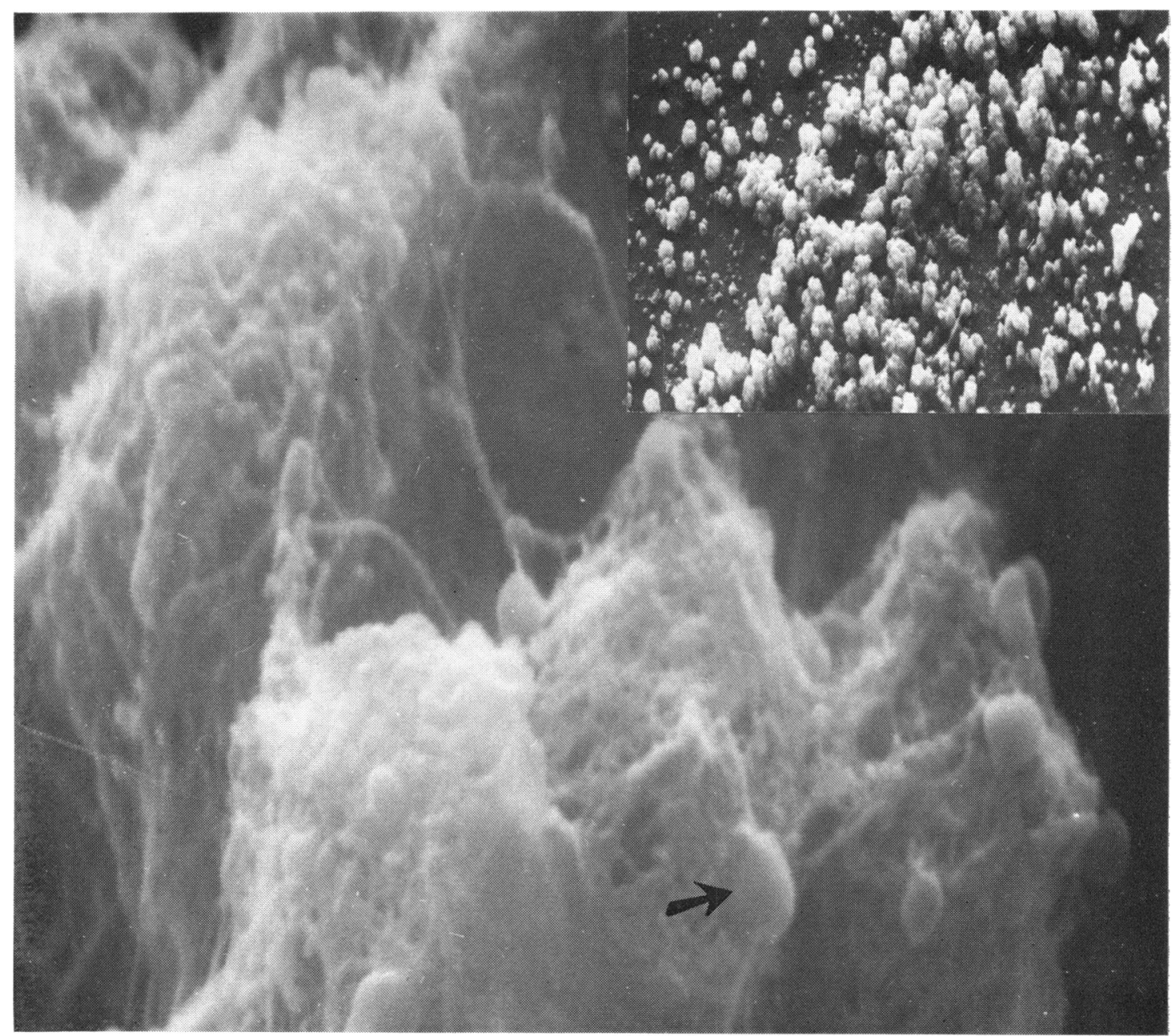

Fig. 3 Anterior surface of the lens in PE disease. Individual vegetations composed of fibrils and ovoid bodies (arrowed). $S E M \times 6100$ from the granular zone (inset). SEM $\times 275$

electron-lucent material are also present. These structures embedded in the surface correspond both in dimensions and in frequency of distribution with the ovoid bodies seen in SEM. A thin layer of PE fibrils lies flat on the surface. They are rarely aggregated in a bush-like arrangement.

Granular Zone.-As in the central zone a thin layer of PE fibrils lies on the superficial capsular lamellae, which are somewhat more compact than the rest of the capsule. This tendency for this surface layer to split away from the rest of the capsule and in some instances to roll up like a scroll is marked (Figs. 5 and 6). Resting on the superficial capsular layer are the vegetations (Fig. 7a). They are composed of intertwined PE fibrils, among which are regularly found a variety of organelles, including pigment granules, mitochondrial elements, and fragments of cytoplasm (Fig. 7b). PE fibrils, which are best studied in the vegetations of the granular and pre-equatorial zones, vary in width from 10 to $40 \mathrm{~nm}$ (the latter being much more frequent), while cross-striations occur at $20 \mathrm{~nm}$ and appear to extend somewhat behind the main body of the fibril, giving it a characteristic smudgy outline. At high magnification it is sometimes possible to determine that the larger PE fibrils are composite, consisting of several subunits which are about $10 \mathrm{~nm}$ wide (Fig. 8).

Pre-equatorial Zone.-Zonular fibres when present in this region are encrusted with accumulations of PE fibrils, especially on their lateral aspects. The 


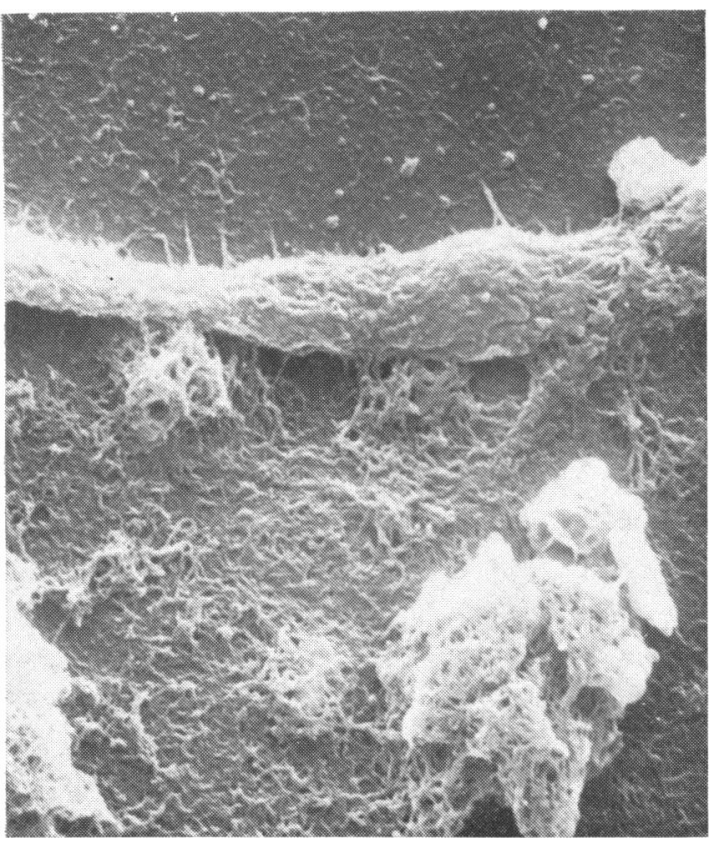

Fig. 4 Anterior surface of the lens in PE disease. Granular zone with vegetations lying on layer of fibrils which has rolled edge. SEM $\times 4010$

zonular fibrils exhibit no intrinsic abnormalities even under high magnification. For the most part PE and zonular fibrils are not intermingled but there are occasional foci in which a degree of admixture occurs. PE fibrils lie on the capsule sometimes as a layer and at other times as vegetations. As in the other zones, numerous organelles are mixed in with PE fibrils. In 3 lenses the presence of sheets of iris pigment epithelial cells adherent to the PE material covering the zonules was noted.

The capsule itself contains numerous large granular inclusions. The characteristic deep, striated or amorphous layer lying next to the epithelium is seen in many sections from this region (Fig. 9). This layer consists of rarefied capsular material, traversed by compact bundles of fibrils which splay out vertically from pits in the underlying epithelium. These vertical fibrils are about $10 \mathrm{~nm}$ wide. Finger-like cytoplasmic processes extend from the epithelial cells into the striated band. It is not possible to determine whether certain organelle-like elements in this layer lie free or are produced by cutting these processes obliquely. The underlying epithelium shows variable vacuolation but no evidence of increase in organelles. Multilaminar basement membrane production is evident in some parts of the amorphous layer, but this is not a regular feature.

\section{HISTOCHEMISTRY}

In haematoxylin and eosin preparations PE material and the capsule proper are stained pink while the striated band has a bluish tinge. Gomori's chrome haematoxylin colours the PE material on the lens surface and the capsular inclusion (Fig. 10) and the striated band a variable dark blue (Fig. 11a). The striations are intensely stained as black lines in vertical sections but as black dots in tangential cuts. The zonular fibres are also blackened by this method.

Congo red dichroism is not present in any of the sections examined. Sections stained with thioflavins $\mathrm{T}$ and $\mathrm{S}$ were examined by ultraviolet light. Green fluorescence is observed in PE material on the anterior surface of the capsule and capsular inclusions (Fig. 10 inset) and in the striated band (Fig. $11 b$ ). The striations but not the intervening capsular material are stained, appearing as bright vertical lines or dots, the appearance depending on whether perpendicular or tangential sections are studied. Zonular fibres are unstained. No fluorescence of the capsular PE materials or inclusions is noted after treating unfixed frozen sections with fluorescein antibody conjugates which had been raised to lambda and kappa immunoglobulin chains. These con-

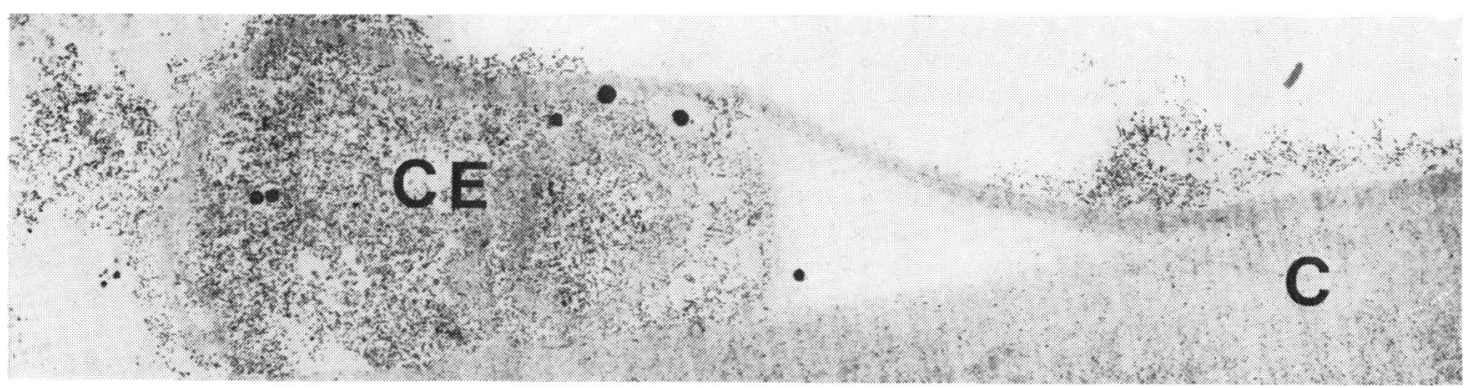

Fig. 5 Granular zone in PE disease. Dehiscence of the capsule (C) has resulted in a scroll-like composite of superficial capsule, PE fitrils and various organelles. Exfoliated capsule (CE). TEM $\times 11600$ 


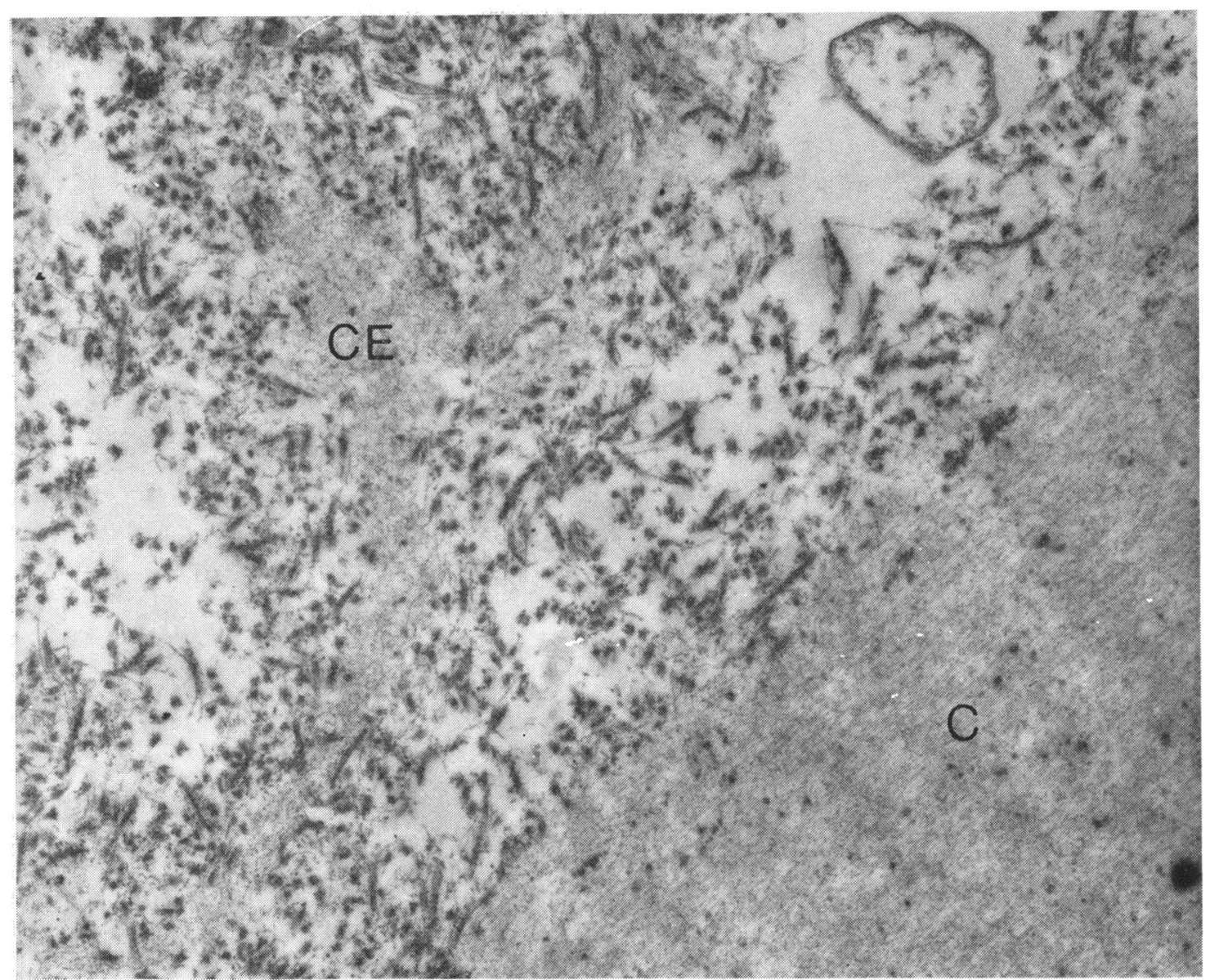

Fig. 6 Detail from Fig. 5 showing exfoliated capsule (CE) with PE fibrils intimately adherent to it. C is main capsule. TEM $\times 26780$

jugates produced intense cytoplasmic fluorescence in plasma cells which were present in bone marrow sections used as controls.

\section{Comments}

The several zones of the anterior lens capsule in PE disease are readily discernible by slit-lamp microscopy. At high magnification the granular zone is seen as fine white dots (the vegetations) on a greyish translucent background layer. The central edge of this layer is often rolled up in a scroll-like fashion. A similar separation of the edges of the central zone is sometimes seen. Indeed, we have personally observed exfoliation along with faint radial striae as one of the earliest biomicroscopic features of this disease. The present TEM and SEM studies indicate that this tendency of the granular and central zones to desquamate is a true dehiscence of the superficial capsule for which the term 'pseudoexfoliation' is clearly inappropriate. Exfoliation of the superficial lens capsule was also noted in the recent TEM studies of Ghosh and Speakman (1972) and of Sugar (1976).

The central zone in PE disease, present in about $90 \%$ of patients with this disorder, was examined with SEM by Benedikt et al. (1973), who found a verrucose surface texture and noted a tendency for the disc edges to curl up. TEM observations by the same authors (Benedikt et al., 1973) indicated that it was composed of typical PE fibrillar material. In the present study the central disc is seen as an even layer of fibrils intermixed with ovoid bodies. TEM correlates of the ovoid bodies indicate that they consist not only of uveal pigment granules, as Davanger and Pederson (1975) observed, but also of various membrane-bound organelles and cell fragments. Their origin from iris pigment epithelial cells is deduced from the appearance of individual 


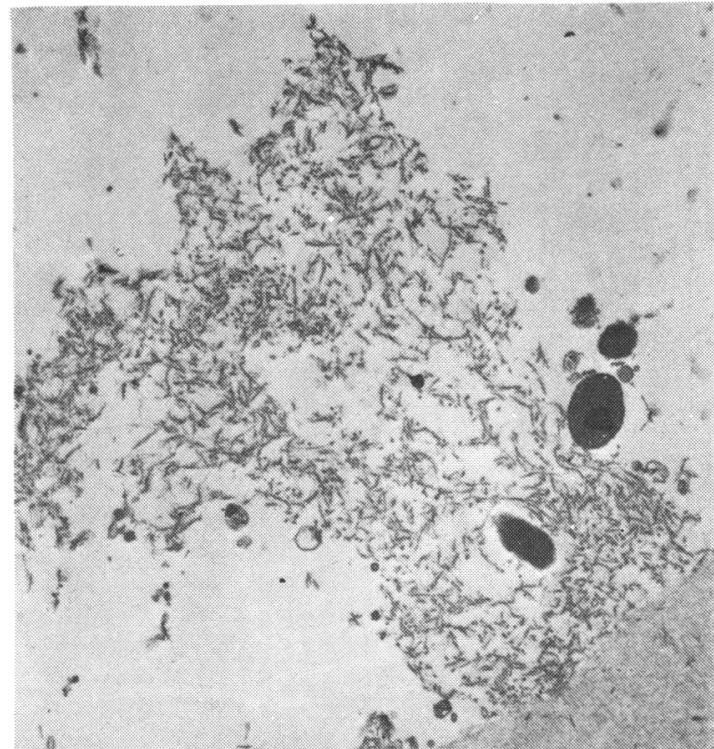

Fig. $7 a \quad A$ vegetation of the granular zone showing admixture of fibrils and organelles. TEM $\times 9530$

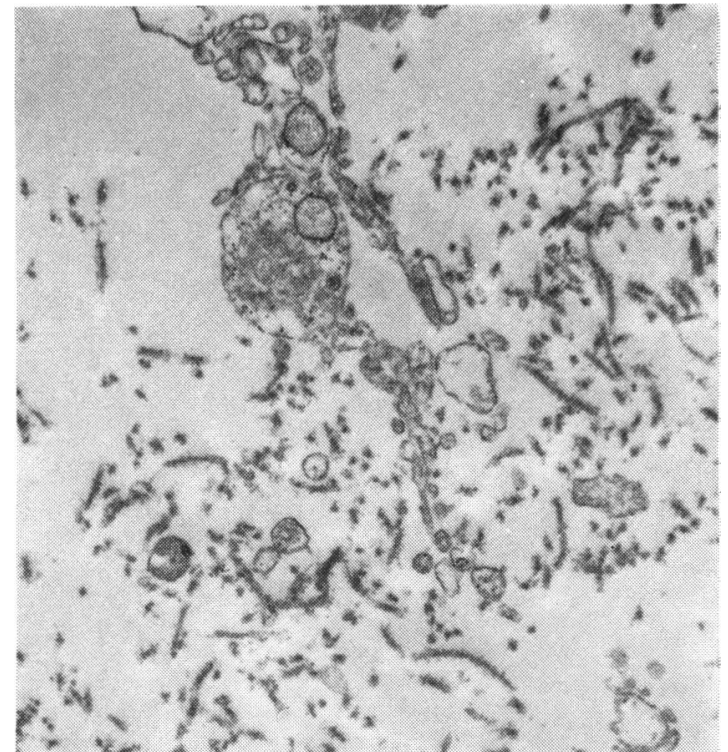

Fig. $7 b$ Detail from Fig. 7a. Cell remnants including organelles lie scattered randomly throughout the vegetation. TEM $\times 18518$

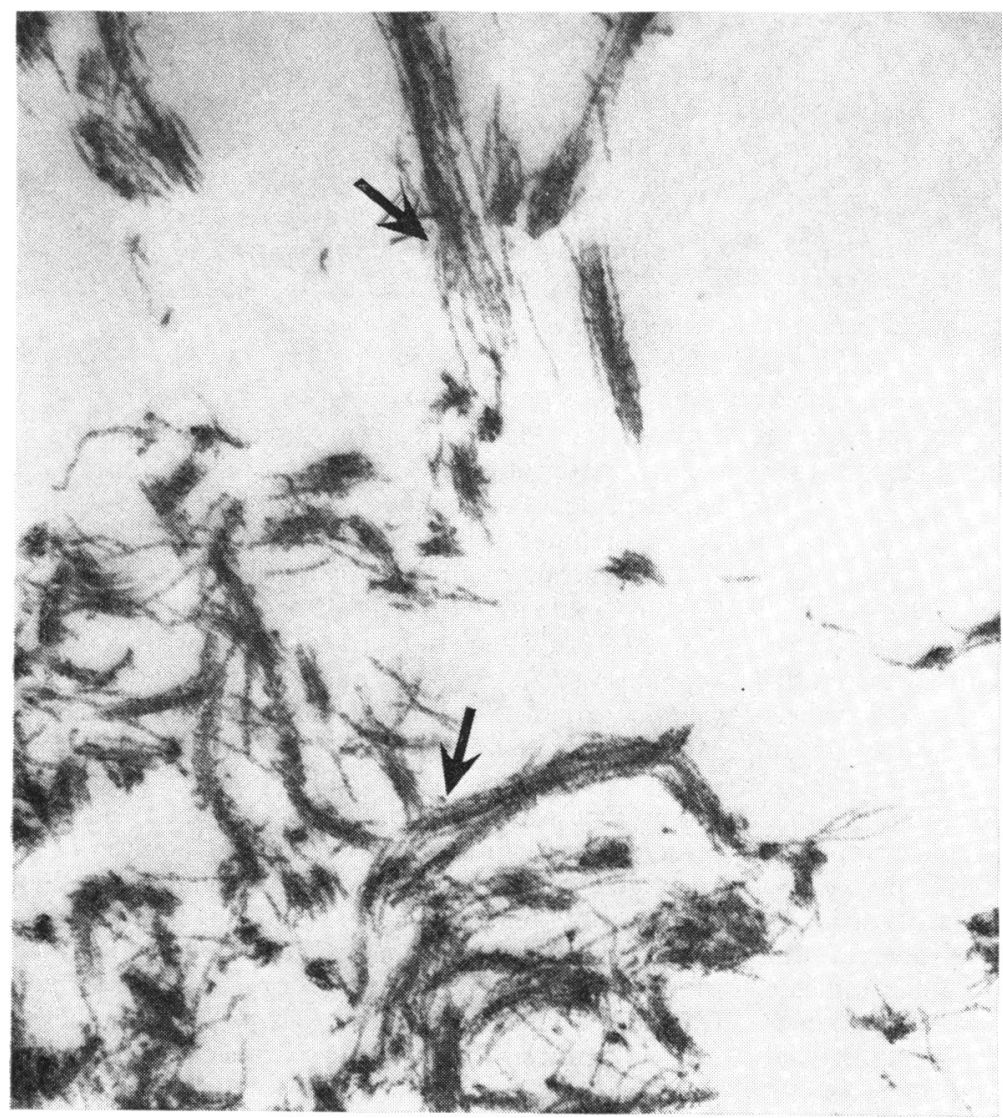

Fig. 8 PE fibrils (composed of filamentous subunits (arrows)). $T E M \times 56130$ 


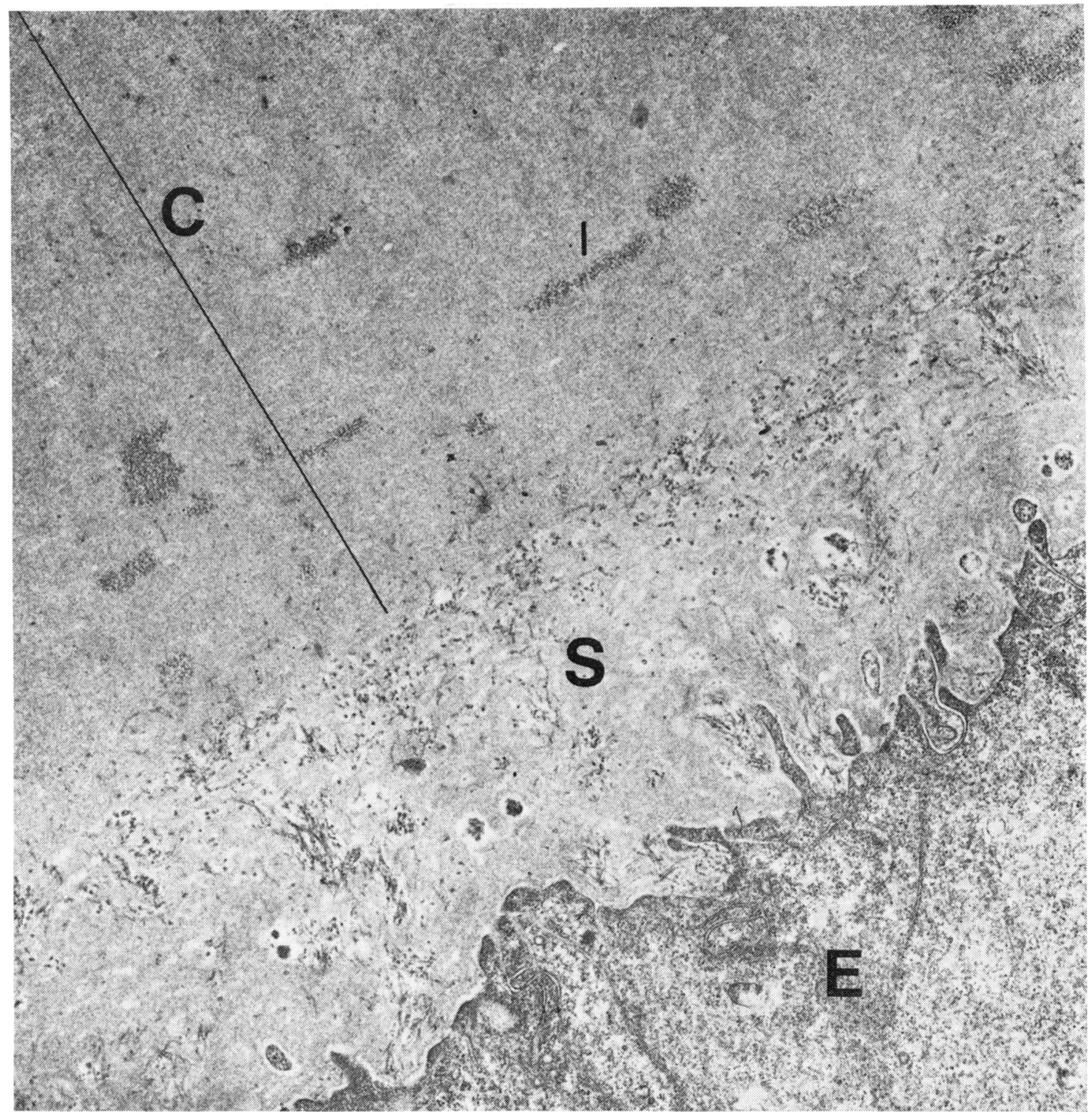

Fig. 9 Pre-equatorial zone in PE disease. Capsule showing bilaminar structure. The capsule proper (C) in which are embedded numerous large granular inclusions (I). In the deep or striated portion (S) vertically orientated bundles of fibrils lie in pits of the underlying epithelial cells (E) many of which exhibit finger-like cytoplasmic processes.

$T E M \times 13855$

or even sheets of pigment cells which are strongly adherent to PE material on the pre-equatorial capsule. In some patients this bonding is so strong that lens delivery normally accomplished with surgical ease in this condition is possible only after overcoming protracted resistance.

The present study indicates that vegetations of the granular zone are also composed of the same elements-fibrils and organelles. There is poor correlation of fibril size when TEM and SEM measurements are compared. Our SEM measurements indicated a diameter of 40 to $60 \mathrm{~nm}$. It is also certain that these measurements are exaggerated by carbon and gold coating. Davanger and Pederson (1975) observed that PE fibrils on the lens are coated with a layer of ruthenium red reactive material, 


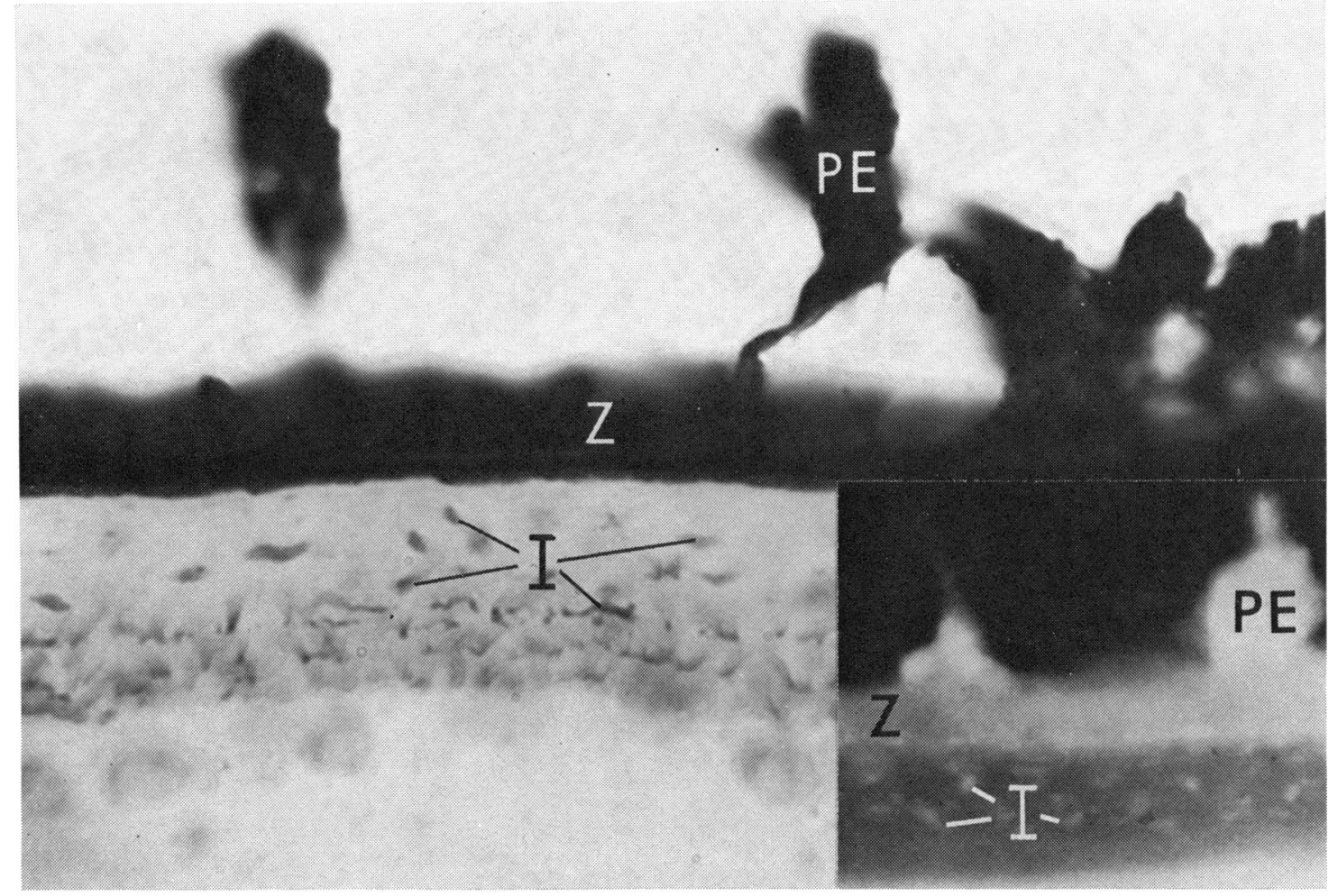

Fig. 10 Pre-equatorial lens capsule. PE material (PE) on the lens capsule, the zonules (Z), and the capsular inclusions (I) are coloured blue-black. Chrome haematoxylin $\times 1120$. Inset pre-equatorial zone showing green fluorescence of PE material (PE) on the lens surface and capsular inclusions $(\mathrm{I})$. Zonules $(\mathrm{Z})$ are unstained. Thioflavin $T \times 673$

presumably mucopolysaccharide, which may further contribute to this discrepancy. PE fibrils seen on TEM were reported to be up to $1 \mu \mathrm{m}$ long by Ashton et al. (1965), whereas those seen here in SEM may be as long as $3 \mu \mathrm{m}$. Dickson and Ramsay (1975) noted that the fibrils composing the vegetations were of a larger diameter $(25 \mathrm{~nm})$ than those found on the surface layer $(15 \mathrm{~nm})$. In the present study no significant difference was noted between the fibril diameter in these two situations. Spiral fibrils on the surface of vegetations noted in SEM by Davanger (1975) were not encountered in the present study.

In spite of the generally acknowledged clinical frequency of lens subluxation in PE disease, the zonules appeared for the most part normal in the present TEM study, though occasional foci of infiltrations with PE fibrils were found. Further study of the zonules in PE disease is clearly indicated, especially near their ciliary attachments, the site at which spontaneous rupture seems to occur preferentially, as noted by Bartholomew (1970).
The amino-acid profile of PE material deposited on the lens was analysed by Ringvold (1973b) and found to be similar to that of amyloid. PE fibrils are generally about four times thicker than those of amyloid, which are usually $10 \mathrm{~nm}$ wide. However, Ringvold and Husby (1973) have shown that PE fibrils found in the iris are composed of several subunits each about $10 \mathrm{~nm}$ wide; a similar observation has been made for PE fibrils on the lens capsule in the present study. Moreover, PE fibrils found in the striated band of the pre-equatorial capsule are all about $10 \mathrm{~nm}$ wide, an observation first made by Davanger (1975) and confirmed by us. On occasion amyloid fibrils also show lateral aggregation, forming fibres about $40 \mathrm{~nm}$ wide as illustrated by Cohen (1968). Davanger (1975) has observed that individual PE fibrils are coated with mucopolysaccharide and lie in a mucopolysaccharide matrix, also resembling amyloid in this respect. Ringvold and Husby (1973) demonstrated that PE fibrils in the iris stained positively with fluorescein-conjugated antibody raised against liver amyloid. They did not, as confirmed in 


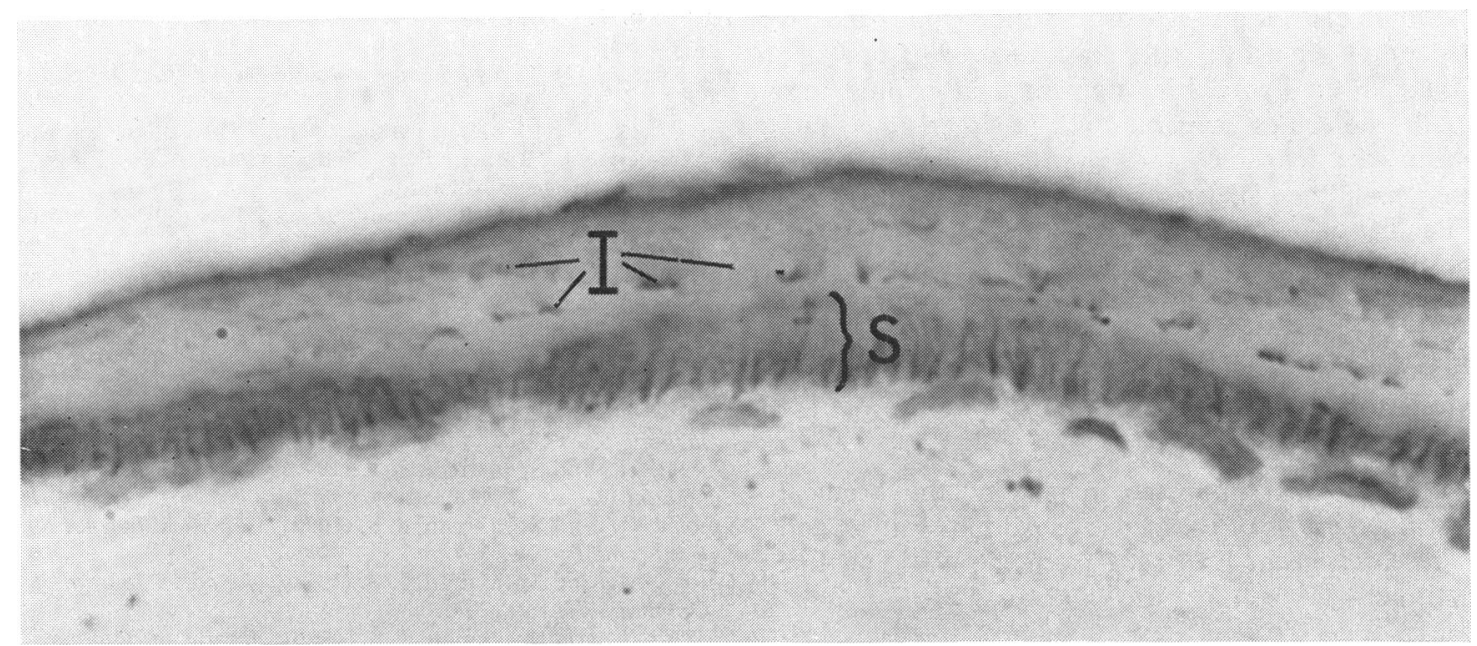

Fig. 11 a Pre-equatorial lens capsule in PE disease. Black vertical striae (S) in deep zone of capsule. Capsular inclusions (I) and zonules on the lens surfaces are also stained. Chrome haematoxylin $\times 1108$

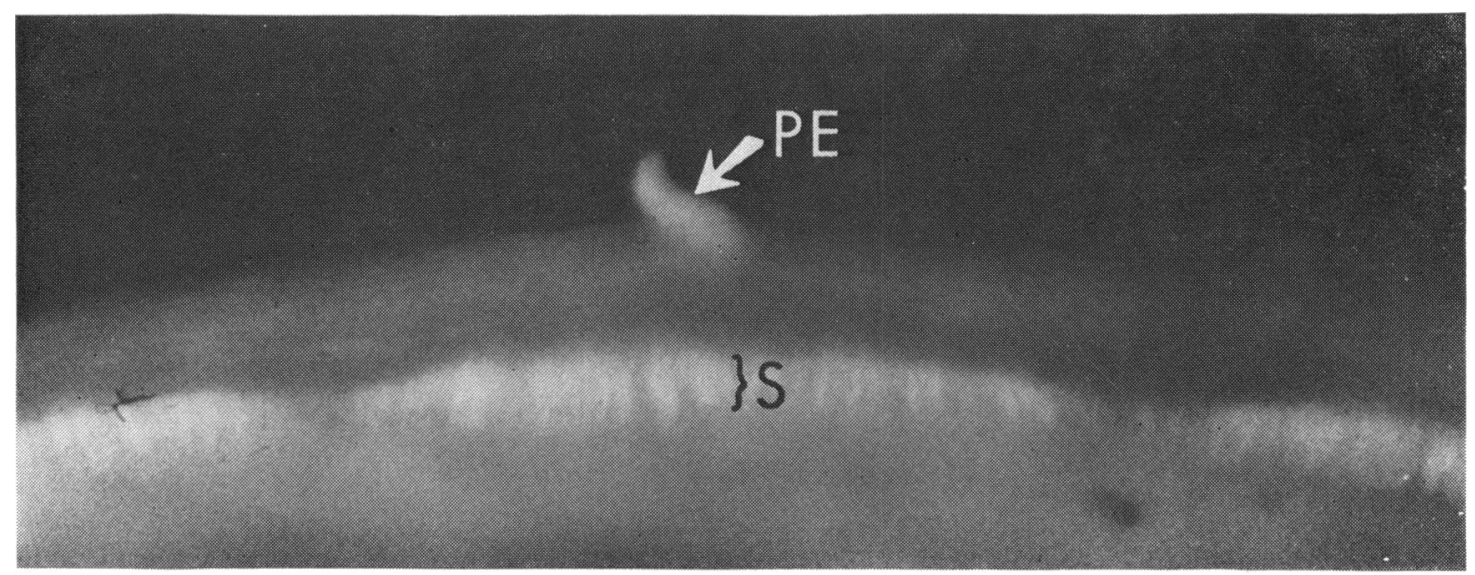

Fig. $11 b$ Pre-equatorial lens capsule in PE disease. Vertical striae (S) in deep laver of capsule and PE material (PE) on the lens surface show green fluorescence in ultraviolet light. Thioflavin $T \times 1108$

the present study, stain with fluorescein conjugates to lambda and kappa chains, so that it would seem unlikely that immunoglobulins are the source of PE fibrils. PE fibrils in the iris were shown by Ringvold and Husby (1973) to exhibit fluorescence in ultraviolet light after staining with the thioflavins $T$ and $S$, we observed a similar reaction with PE fibrils on and in the lens capsule. It must be admitted, however, that thioflavin fluorescence, while typical of amyloid, lacks histochemical specificity.

Thus on the one hand PE and amyloid fibrils share a number of ultrastructural, biochemical, and histochemical properties. On the other hand, Congo red dichroism, generally regarded as the most critical histochemical test for amyloid, is not shown by PE fibrils in the iris (Ringvold, 1973b) or as note here in relation to the anterior lens capsule.

Glenner et al. (1972) consider amyloid to result from enzymatic (lysozomal) fragmentation of longchain proteins (often immunoglobulins) into polypeptides, which polymerise spontaneously into an antiparallel beta-pleated sheet configuration, of which Congo red dichroism is probably the histochemical counterpart. They have thus founded the concept that under certain conditions, a pathogenic protein can be formed by proteolysis of an innocuous precursor. On the basis of the histochemical and electron microscopic findings in the present study of PE disease it may be suggested that the preequatorial epithelial cells synthesise long-chain 
zonule-like proteins, which are then converted by lysozomal enzymes (perhaps derived from the damaged or dying iris pigment epithelium) into small molecules capable of polymerising both within the capsule and in relation to nearby aqueous-bathed structures.

Peeling or exfoliation of the surface capsule in PE disease is not readily explained from the information available. The numerous organelles scattered over the anterior lens capsule in PE disease suggest that lysozomal enzymes could also play a role in exfoliation.

This study was supported in part by the Medical Research Service of the Veterans Administration and by research grant EY 01602 from the National Eye Institute.

\section{References}

Ashton, N., Shakib, M., Collyer, R., and Blach, R. (1965). Investigative Ophthalmology, 4, 141.

Bartholomew, R. S. (1970). British Journal of Ophthalmology, 54, 744.

Bartholomew, R. S. (1971). British Journal of Ophthalmology, 55, 693.

Benedikt, O., Göttinger, W., and Auböck, L. (1973). Acta Ophthalmologica, 51, 211.

Bertelsen, T. I., Drablos, P. A., and Flood, P. R. (1964). Acta Ophthalmologica, 42, 1096.
Bertelsen, T. I., and Ehlers, N. (1969). Acta Ophthalmologica, 47, 476.

Busacca, A. (1928). Albrecht v. Graefes Archiv für Ophthalmologie, 119, 135 .

Cohen, A. S. (1968). Amyloidosis, p. 154. Edited by E. Mandema, L. Ruinen, F. H. Scholten, and A. S. Cohen. Excerpta Medica: Amsterdam.

Dark, A. J., Streeten, B. W., and Jones, D. (1969). Archives of Ophthalmology, 82, 815.

Davanger, M., and Pederson, O. (1975). Acta Ophthalmologica, 53, 3.

Davanger, M. (1975). Acta Ophthalmologica, 53, 809.

Dickson, H. D., and Ramsay, M. S. (1975). Canadian Journal of Ophthalmology, 10, 148.

Dvorak-Theobald, G. (1954). American Journal of Ophthalmology, 37, 1.

Ghosh, M., and Speakman, J. S. (1972). Canadian Journal of Ophthalmology, 7, 413.

Ghosh, M., and Speakman, J. S. (1973). Canadian Journal of Ophthalmology, 8, 394.

Ghosh, M., and Speakman, J. S. (1974). Canadian Journal of Ophthalmology, 9, 289.

Ghosh, M., and Speakman, J. S. (1976). Archives of Ophthalmology, 94, 1757.

Gifford, H. (1957). Transactions of the American Ophthalmological Society, 55, 189.

Glenner, G. G., Terry, W. D., and Isersky, G. (1973). Seminars in Hematology, 10, 65.

Ringvold, A. (1973a). Acta Ophthalmologica, 51, 411.

Ringvold, A. (1973b). Experimental Eye Research, 15, 37.

Ringvold, A., and Husby, G. (1973). Experimental Eye Research, 17, 289.

Sugar, H. S. (1976). Survey of Ophthalmology, 21, 59.

Vannas, A. (1969). Acta Ophthalmologica, Supplement, 105.

Vogt, A. (1925). Klinische Monatsblätter für Augenheilkunde, 75,1 . 